\title{
REVISITING DRIVER BEHAVIOR AT UNSIGNALIZED INTERSECTIONS: TIME OF DAY IMPLICATIONS FOR TWO-WAY LEFT TURN LANES (TWLTL)
}

\author{
Sahar Nabaee, Derek Moore, \& David Hurwitz \\ Oregon State University \\ Corvallis, Oregon, USA \\ Email: david.hurwitz@oregonstate.edu
}

\begin{abstract}
Summary: A novel procedure was developed and validated for the accurate observation of naturalistic driver gap acceptance behavior at unsignalized intersections. Specifically, two-way stop-controlled intersections with a two way left turn lane (TWLTL) on the major road were examined. Three intersections were included as experimental locations. A sample size was collected of approximately 875 minor street vehicles which were exposed to over 2400 individual gaps. Characteristics such as gender, approximate age, vehicle type, presence of a queue behind the lead vehicle, and presence of passengers in the vehicle were collected as a function of the time of day (TOD). This work provides updated measures for the accepted gap as TOD varies, as well as exploring how accepted gaps are related to the wait time of a vehicle at the stop line.
\end{abstract}

\section{INTRODUCTION}

Two-way stop-controlled (TWSC) intersections are one of the most common intersection types in the United States (Kittleson and Vandehey, 1991). This type of intersection control is most commonly applied where roads of different functional classification intersect (Pollatschek et al. 2001). At TWSC intersections priority is provided to the major approach (es) (no traffic control present), while stop signs control vehicular movements on minor approach (es). Drivers on the minor approach should yield the right of way to traffic on the major road and proceed into the intersection only after a full stop is performed and when there is a large enough gap between two successive vehicles on the major road to safely execute the maneuver of interest, in such manner that the traffic stream on the mainline remains unaffected (HCM, 2000).

\section{Gap Acceptance/Critical Gap}

When a driver arrives at the stop line on the minor approach to a TWSC intersection, they need to decide when to execute a maneuver based on right of way hierarchy as well as the availability and distributions of the major road gaps (HCM 2000). Due to the important role that personal driver behavior plays in confronting the conflicting traffic, the capacity and level of service analysis for TWSC intersections are more complex than that of intersections with higher levels of control (Kittleson and Vandehey, 1991).

The critical gap is a principal parameter of the capacity analysis at TWSC intersections. Previous studies defined the critical gap as the size of the gap for which half of all traffic will reject larger gaps while half will accept smaller gaps (Drew, 1968). More recent studies have augmented this general definition by postulating that those gaps large enough to be accepted by almost all drivers (approximately 12s to 15s) provide little meaningful information about drivers' gap 
acceptance behavior and should not be used in the analysis (Gattis and Low, 1999). The most commonly accepted definition for critical gap is the minimum usable gap accepted by the minor approach drivers (Roess et al. 2004). This definition assumes all the gaps that are equal to or greater than the critical gap will be accepted and all the smaller gaps will be rejected (HCM, 2000). It cannot be overstated that gap acceptance behavior is highly dependent on the driver characteristics and preferences. Therefore, homogeneous behavior from the all drivers at all times is not realistic.

In fact, drivers on minor approaches have shown a tendency to accept a gap when "the benefit from entry is greater than the associated risk" (Pollatschek et al. 2002). When the waiting time exceeds the drivers' expectation and tolerance limit, they will accept higher levels of risk associated with smaller gaps. It is somewhat unclear in the literature if drivers accurately perceive the increased risks associated with the acceptance of these smaller gaps. After a certain wait time threshold, drivers might even accept gaps shorter than gaps that had previously been rejected (Xiaoming et al. 2007).

It has also been shown that intersection geometries can greatly influence gap acceptance behavior. Hamed et al. (1997) studied left turn maneuvers at stop-controlled T-intersections and found that the presence of a median with an exclusive left-turn lane on the major approach resulted in smaller critical gaps. According to the Highway Capacity Manual (HCM) 2000, the presence of a storage area on the major road that is wide enough to accommodate left turning vehicles, allows for two-stage gap acceptance, resulting in the entire left turn maneuver being considered as two separate stop controlled maneuvers. Thus, Intersections with a TWLTL on the major approach have improved safety and capacity, in comparison with undivided major approaches (HCM, 2000). In fact, a continuous TWLTL might result in up to 35\% decrease in total crashes, 30\% decrease in delay, and 30\% increase in capacity (Access Management Manual, 2003). Hamed et al. (1997) also studied the gaps at different times of the day and found that during off-peak periods, gaps are larger and more stable, making it easier for the drivers to perform a left turning maneuver, resulting in shorter wait times during off-peak periods than for peak periods.

Much of the previous gap acceptance research has concerned itself with the safety implications of selecting smaller gaps. This is likely related to the seriousness of crashes resulting from the selection of inappropriately small gaps. Relatively less attention has been paid to the capacity implications of gap acceptance behavior.

The focus of this study was to contribute to the literature by focusing on a geometric configuration that has received relatively less attention than others (T-intersections with a TWLTL on the major approach) as well as to consider factors that have not previously been considered as influencing gap acceptance behavior. These factors included: approximate driver age, gender, vehicle type, presence of passengers, and queue size on the waiting time and the gaps selected by drivers as the time of day varied. 


\section{METHOD}

This study was concerned with the acquisition and analysis of naturalistic driver behavior as vehicles performed right or left turning movements at a two-way stop-controlled intersection from the minor to the major approach. A software package was developed collaboratively between the University of Massachusetts Amherst (UMass Amherst) and Oregon State University (OSU) to collect the naturalistic field data and aid in the analysis.

\section{Locations}

Experimental locations were restricted to T-intersections (three approaches intersecting at approximately a 90 degree angle) in Corvallis and Albany, Oregon. This configuration resembles that of many driveways experiencing high volumes of traffic and allowed for efficient evaluation of gap acceptance behavior by eliminating the complexities associated with opposing traffic at regular four-leg intersections. To eliminate the effects of different geometric characteristics, locations with similar configurations were selected. Specifically, study intersections have exclusive left turn and right turn lanes on the minor approach, while the major roadway has a TWLTL separating single lanes of opposing traffic. This configuration is fairly common in Oregon. Compared to other intersection configurations, less effort has been expended determining how this affects gap acceptance behavior (Figure 1). By controlling for the physical characteristics of the intersections, factors such as the distance travelled by a vehicle making a turning movement, posted speed limits, and available sight distance remained relatively consistent between experimental locations. This allows for the aggregation of data collected at each location increasing the sample size and statistical significance of the results. It was desired to capture gap acceptance behavior throughout the day to determine if the critical gap is influenced by time of day. Data collection intervals were established to break the day into four sections and data was collected at each location during each time interval.

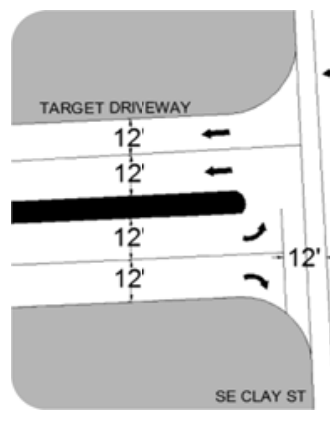

\section{Software Package}

The software package, developed as a collaborative effort between the University of Massachusetts Amherst and Oregon State University, is an Access based program developed specifically for collecting gap acceptance information. While running the program on a laptop, the Gap Acceptance Processing System (GAPS) allowed for accurate and efficient data collection in the field. Figure 2 shows the graphical user interface (GUI) visible to the researcher when collecting gap data, illustrating a vehicle making a right turn and the corresponding 
keystroke. The software allows researchers to collect gap availability and acceptance as well as many other driver, intersection and environmental characteristics.

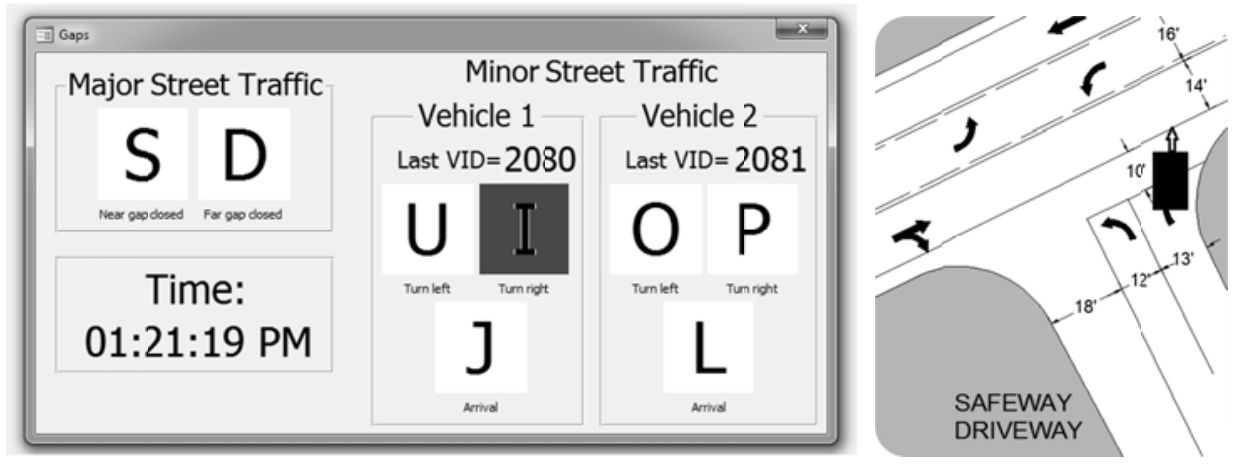

Figure 2. Screenshot while collecting data with the GAPS program (left) and corresponding vehicle movement (right)

\section{Procedure}

The data collection protocol required pairs of researches in the field to complete the observations with the requisite degree of accuracy. One researcher was responsible for running the GAPS software on the laptop while the other would capture detailed information about the drivers and vehicles approaching the stop sign on the minor street. This detailed information included gender, approximate age, presence of passengers, queue size and vehicle type, which was input into the program database at the completion of each site visit. Once all data had been collected, macros imbedded in the GAPS software reduced the data into a file easily transferred and analyzed in Microsoft Excel.

\section{Validation of Data Collection Procedure}

To ensure the accuracy of the field observations acquired with the use of the GAPS software, a high-definition video camera was used to simultaneously record one of the stop controlled intersections while manual data collection was taking place. 50 minor street vehicles were captured in conjunction with the mainline gaps. The video was reduced in the office on high definition televisions in slow motion to provide accurate information about the exact time (accurate to a hundredth of a second) vehicles arrived at the intersection, initiated a right or left turn, and the length of available gaps in mainstream traffic. Data obtained from manual data collection was compared to the video transcription, which was assumed to be accurate. This initial validation was concerned with three measures vital to the analysis of the database. It was initially verified that the number and order of vehicles entering the intersection on the minor approach matched the video exactly. A comparison of wait time, defined here as the time between the vehicle reaching the stop line and initiating the turning movement, proved to be reliable at a $90 \%$ threshold with an average difference of 1.38 seconds. Additionally, each accepted gap greater than 15 seconds was removed from the analysis. The comparison of the accepted gaps used in analysis revealed that approximately $80 \%$ of the accepted gaps observed manually were within one second of the actual accepted gaps acquired from the video. 


\section{RESULTS}

To perform analyses based on the characteristics of the drivers and vehicles, it was essential to identify any trends or shifts in these characteristics seen throughout the day. Table 1 displays the percentage of each demographic as it varies throughout the day. In some cases approximate age and gender could not be accurately determined, in which case they were identified as "Not Sure". By visual inspection, there does not appear to be any significant shifts in the population demographics that could potentially affect the analysis.

Table 1. Sample Demographics

\begin{tabular}{ccccccc}
\hline & & $7: 00 \mathrm{am}-10: 00 \mathrm{am}$ & $10: 00 \mathrm{am}-1: 00 \mathrm{pm}$ & $1: 00 \mathrm{pm}-4: 00 \mathrm{pm}$ & $4: 00 \mathrm{pm}-7: 00 \mathrm{pm}$ & Combined \\
\hline \multirow{3}{*}{$\begin{array}{c}\text { Age } \\
\text { Category }\end{array}$} & Teen & $19(9 \%)$ & $25(12 \%)$ & $42(19 \%)$ & $41(18 \%)$ & $127(15 \%)$ \\
& Adult & $172(78 \%)$ & $158(73 \%)$ & $129(60 \%)$ & $157(70 \%)$ & $616(70 \%)$ \\
& Elder & $27(12 \%)$ & $22(10 \%)$ & $35(16 \%)$ & $19(9 \%)$ & $103(12 \%)$ \\
\multirow{5}{*}{ Gender } & Not Sure & $3(1 \%)$ & $10(5 \%)$ & $10(5 \%)$ & $7(3 \%)$ & $30(3 \%)$ \\
& Male & $106(48 \%)$ & $91(42 \%)$ & $102(47 \%)$ & $114(51 \%)$ & $413(47 \%)$ \\
& Female & $115(52 \%)$ & $117(55 \%)$ & $109(50 \%)$ & $106(47 \%)$ & $447(51 \%)$ \\
& Not Sure & $0(0 \%)$ & $7(3 \%)$ & $5(3 \%)$ & $4(2 \%)$ & $16(2 \%)$ \\
\hline
\end{tabular}

A very important characteristic of gap acceptance behavior is the amount of time spent waiting for an acceptable gap. Figure 3 illustrates the relationship between the accepted gap and wait time for approximately 875 cars, which is comparable to relationships seen in previous research (Xiaoming et al. 2007). To gain a better understanding of how this relationship changes based on demographic variables, similar plots were created for individual characteristics, however these plots revealed little information due to the sample size collected. It should be noted that the plot on the left panel displays the entire database, but accepted gaps of greater than 15 seconds have been eliminated from the analysis in the right panel.
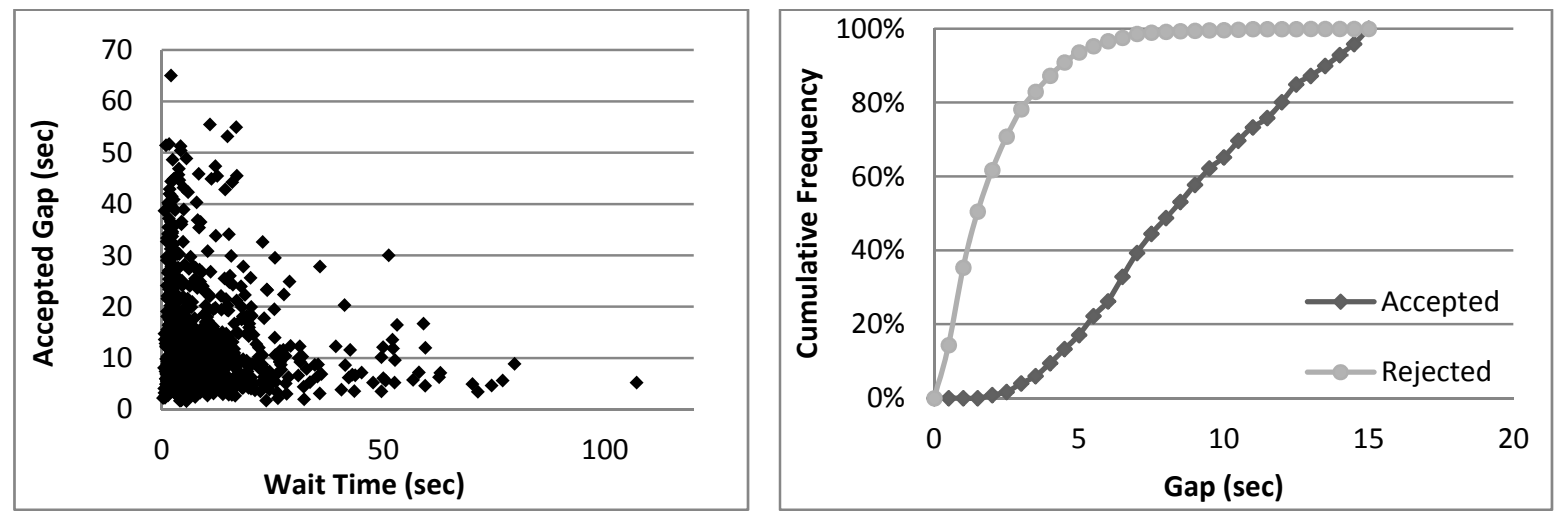

Figure 3. Accepted gaps plotted against wait time (left) and cumulative frequency of both rejected and accepted gaps (right)

A visual inspection of the data presented in the left panel of Figure 3 seems to show that the relationship between wait time and accepted gap generally takes the shape of a negative 
exponential distribution. From the raw data it appears that as the wait time increases, shorter gaps are more commonly accepted than longer ones.

From the total right and left turn data presented in Table 2, it appears that later in the day, the average gap accepted by drivers to perform right and left turns decreases by up to 9 and 21 percent, respectively. When considering age categories, teen drivers usually tend to accept shorter gaps than their older counterparts. However, an ANOVA test did not provide evidence of a significant difference for either left or right turn maneuvers among age categories. It is also apparent that there is little shift in left turn gap acceptance behavior among older drivers (no statistical difference at 95\%), while teen drivers show a relatively larger shift in their behavior at different times of the day (statistically significant at 95\%).

Table 2. Average Accepted Gaps (seconds)

\begin{tabular}{|c|c|c|c|c|c|c|c|c|c|c|c|c|}
\hline & \multicolumn{2}{|c|}{ Total } & \multicolumn{2}{|c|}{ Male } & \multicolumn{2}{|c|}{ Female } & \multicolumn{2}{|c|}{ Teen } & \multicolumn{2}{|c|}{ Adult } & \multicolumn{2}{|c|}{ Elder } \\
\hline & Mean & $+/-\mathrm{CI}$ & Mean & $+/-\mathrm{CI}$ & Mean & $+/-\mathrm{CI}$ & Mean & $+/-\mathrm{CI}$ & Mean & $+/-\mathrm{CI}$ & Mean & $+/-\mathrm{CI}$ \\
\hline \multicolumn{13}{|l|}{ Left Turn } \\
\hline 7:00am-10:00am & 8.45 & 0.93 & 8.02 & 1.33 & 8.91 & 1.39 & 11.18 & 2.60 & 7.98 & 1.02 & 8.71 & 4.34 \\
\hline 10:00am-1:00pm & 8.76 & 0.72 & 8.90 & 1.27 & 8.75 & 0.92 & 7.79 & 3.24 & 8.92 & 0.80 & 8.96 & 3.29 \\
\hline 1:00pm-4:00pm & 7.22 & 0.72 & 6.64 & 0.94 & 7.72 & 1.13 & 7.46 & 1.92 & 7.10 & 0.92 & 7.21 & 1.81 \\
\hline 4:00pm-7:00pm & 7.84 & 0.74 & 7.70 & 1.10 & 7.76 & 1.03 & 6.24 & 1.08 & 7.94 & 0.92 & 8.50 & 2.64 \\
\hline \multicolumn{13}{|l|}{ Right Turn } \\
\hline 7:00am-10:00am & 9.55 & 0.81 & 9.07 & 1.12 & 10.01 & 1.12 & 8.32 & 3.28 & 9.84 & 0.90 & 7.64 & 4.45 \\
\hline 10:00am-1:00pm & 8.88 & 0.89 & 9.57 & 1.28 & 8.46 & 1.31 & 8.71 & 4.43 & 9.34 & 1.00 & 6.87 & 3.06 \\
\hline 1:00pm-4:00pm & 8.66 & 0.78 & 8.74 & 1.34 & 8.66 & 1.00 & 8.77 & 1.92 & 8.47 & 1.07 & 9.35 & 1.79 \\
\hline 4:00pm-7:00pm & 8.66 & 0.66 & 8.57 & 0.83 & 8.77 & 0.98 & 7.42 & 1.56 & 8.72 & 0.75 & 10.48 & 2.52 \\
\hline
\end{tabular}

Two-sample t-tests were performed to compare the mean accepted gaps based on the presence of passengers in the car, as well as by presence of a queue behind the turning vehicle. For left turn maneuvers, the results showed a trend toward significantly shorter accepted gaps in the presence of passengers $(\mathrm{P}$-value $=0.053)$. Also, drivers with vehicles waiting behind them accepted significantly shorter gaps than those without $(\mathrm{P}$-value $=0.005)$. Although the same trends for both comparisons were observed for right turns, the differences were not found to be significant.

\section{DISCUSSION}

The findings of this research are generally consistent with previous research on this topic. Research by Xiaoming et al. (2007) identified wait time and the length of queue on the minor approach as factors affecting gap acceptance behavior, with wait time being the most influential. Similarly, Hamed et al. (1997) found that the expected wait time increases as the length of available gaps decreases. They also found that gap acceptance behavior is not uniform throughout the day.

This research effort was intended to develop and test a novel data collection tool for the purposes of quickly and accurately collecting gap acceptance data at TWSC intersections. In doing so, we 
contributed to the understanding of how and why drivers select gaps at TWSC intersections with a TWLTL on the major road. The following conclusions were reached:

- The GAPS software provides reasonably accurate measurements of minor street vehicle's wait time and accepted gaps, providing means for consistent and reliable data collection.

- Evidence suggests that later in the day, drivers accept shorter mean gaps while performing right and left turns.

- It appears that the development of a queue behind the left turning vehicle decreases the accepted gaps by 1.17 seconds with statistical significance. Also, presence of passengers influences the gap acceptance behavior and decreases the accepted gaps by 0.85 seconds with statistical significance.

These observations are critical in our ability to correctly model both capacity and safety at unsignalized intersections.

\section{ACKNOWLEDGMENT}

The research team would like to recognize Dr. Michael A. Knodler and GRA Steven Tupper at UMass Amherst for their efforts in refining the software package and the data collection protocols. Additionally, URA Brennan Burbank and GRA Halston Tuss at OSU contributed to data collection and validation. Without the hard work of these individuals this project would not have been a success.

\section{REFERENCES}

Drew, D. R. (1968). Traffic Flow Theory and Control. New York: McGraw-Hill.

Gattis, J.L., \& Low, S.T. (1999). Gap Acceptance at Atypical Stop-Controlled Intersections. Journal of Transportation Engineering, 125(3), 201-207.

Hamed, M. M. , Easa, S. M., \& Batayneh, R.R. (1997). Disaggregate Gap-Acceptance Model For Unsignalized T-Intersections. Journal of Transportation Engineering, 123(1), 36-42.

Kittleson, W.K., \& Vandehey, M.A., (1991). Delay Effects on Driver Gap Acceptance Characteristics at Two-Way Stop-Controlled Intersections. Transportation Research Record, 1320, 154-159.

Pollatschek, M.A., Polus, A., \& Livneh, M. (2002). A decision model for gap acceptance and capacity at intersections. Transportation Research Part B, 36(7), 649-663.

Roess, R.P., Prassas, E.S., \& McShane, W.R. (2004). Traffic Engineering (Third ed.). Upper Saddle River: Pearson prentice Hall.

Transportation Research Board of The National Academies. (2003). Access Management Manual. Washington, D.C.: Transportation Research Board of The National Academies.

Transportation Research Board of The National Academies. (2000). Highway Capacity Manual. Washington, D.C.: Transportation Research Board of the National Academies.

Zhong Xiaoming, Zhu Xinzheng, Zhang Yong, \& LIU Xiaoming. (2007). Left-turn Gap Acceptance Behavior of Tee Type of Unsignalized Intersection. Paper presented at the International Conference on Transportation Engineering (ICTE) 2007 Chengdu, China. 